\title{
DISTINGUISHING BARE QUARK STARS FROM NEUTRON STARS
}

\author{
P. JAIKUMAR ${ }^{1 * \dagger}$, C. GALE ${ }^{1}$, D. PAGE ${ }^{2}$, AND M. PRAKASH ${ }^{3}$ \\ ${ }^{1}$ Physics Department, McGill University, Montréal, Québec H3A 2T8, Canada. \\ ${ }^{2}$ Instituto di Astronomia, UNAM, Mexico D.F. 04510, Mexico. \\ ${ }^{3}$ Department of Physics \&S Astronomy, SUNY at Stony Brook, Stony Brook, NY
} 11794, USA.

\begin{abstract}
Observations to date cannot distinguish neutron stars from self-bound bare quark stars on the basis of their gross physical properties such as their masses and radii alone. However, their surface luminosity and spectral characteristics can be significantly different. Unlike a normal neutron star, a bare quark star can emit photons from its surface at super-Eddington luminosities for an extended period of time. We present a calculation of the photon bremsstrahlung rate from the bare quark star's surface, and indicate improvements that are required for a complete characterization of the spectrum. The observation of this distinctive photon spectrum would constitute an unmistakable signature of a strange quark star and shed light on color superconductivity at stellar densities.
\end{abstract}

\section{Introduction}

A normal neutron star (NS), composed mainly of neutron matter, is bound by gravitational forces. On the other hand, a strange quark matter (SQM) star, consisting of three-flavor $(u, d, s)$ quark matter up to its surface, can be self-bound due to strong interactions alone. This makes the pressure at the star's surface vanish at a supra-nuclear baryon density. In the context of the MIT bag model with first order corrections due to gluon exchange, the baryon density $n_{B}$ at which the pressure vanishes is ${ }^{1}$

$$
n_{B}(P=0)=\left(\frac{4 B}{3 \pi^{2 / 3}}\right)^{3 / 4}\left(1-\frac{2 \alpha_{c}}{\pi}\right)^{1 / 4}
$$

\footnotetext{
*Work presented at the 26th annual Montreal-Rochester-Syracuse-Toronto conference (MRST 2004) on high energy physics: From quarks to cosmology, May 12 - 14 (2004) Concordia University, Montréal, QC, Canada

†electronic address: jaikumar@hep.physics.mcgill.ca
} 
where $B$ is the bag constant and $\alpha_{c}=g_{c}^{2} /(4 \pi)$ is the quark-gluon coupling constant. For typical values of $B$ and $\alpha_{c}{ }^{2}$, the baryon density at vanishing pressure is about 2 to $3 n_{0}$, where $n_{0}=0.16 \mathrm{fm}^{-3}$ is the saturation density of nuclear matter.

Because of differences in the cause for binding, the mass versus radius relations of NS and SQM stars differ significantly, although in the range of masses $\left(1<M / M_{\odot}<2\right)$ observed to date ${ }^{3}$, the calculated radii are similar $(R \sim 10$ to $15 \mathrm{~km})$. This makes it difficult to distinguish these two classes on the basis of their gross physical properties alone. However, the light curves of NS and SQM stars, determined by surface photon emission, can be very different. At the surface of a SQM star, the density drops abruptly to zero within a distance of the order of a few fm, and charge neutrality requires an electron concentration $n_{e} / n_{B}$ of $10^{-4}$ to $10^{-3}$. These electrons at the surface are bound by electrostatic interaction to quark matter, with radial electric fields whose magnitude $\left(\sim 5 \times 10^{17} \mathrm{~V} \mathrm{~cm}^{-1}\right)$ can exceed the critical value for electron-positron pair production from the QED vacuum.

Recently, it has been pointed out that thermal emission from the bare surface of a strange quark star, due to $e^{+} e^{-}$pair production and subsequent annihilation to photons, can produce luminosities well above the Eddington limit $\left(\sim 10^{38} \mathrm{erg} \mathrm{sec}^{-1}\right)$ for extended periods of time, from about a day to decades ${ }^{4}$. The spectrum of photons is different from that of a normal cooling neutron star $(30<E / \mathrm{keV}<500$ instead of $0.1<E / \mathrm{keV}<2.5)$. Identifying this distinctive spectrum is well within the capabilities of the INTEGRAL satellite ${ }^{5}$ launched toward the end of 2002.

\section{Bremsstrahlung photons from the surface of a bare quark star}

In addition to $e^{+} e^{-}$pair production at the surface, photons are emitted from the bremsstrahlung process $e^{-} e^{-} \rightarrow e^{-} e^{-} \gamma$ in the electron layer, termed the "electrosphere". We report a calculation of the corresponding emissivity in the soft photon limit and compare its magnitude to those from pair-production and blackbody emission. In addition, we characterize the spectrum by its mean energy and quantify deviations from a blackbody spectrum. Our results apply to the case in which there is a charge neutralizing layer of electrons at the surface, including superconducting phases of quark matter ${ }^{6}$.

As the surface temperature falls below $10^{10}$ to $10^{11} \mathrm{~K}(\sim(1-10) \mathrm{MeV})$ within tens of seconds of a compact star's birth, electrons quickly settle 
into a degenerate Fermi sea, and their Fermi momentum far exceeds their rest mass, making it a relativistic degenerate system. In the plane-parallel approximation for the layer of the electropshere, the electron chemical potential as a function of distance $z$ from the quark surface is given by ${ }^{7}$

$$
\mu_{e}(z)=\frac{\mu_{e}(0)}{1+z / H} ; \quad H=\frac{\hbar c}{\mu_{e}(0)} \sqrt{\frac{3 \pi}{2 \alpha}}=501.3\left(\frac{10 \mathrm{MeV}}{\mu_{e}(0)}\right) \mathrm{fm},
$$

where $\alpha=e^{2} / 4 \pi$ is the fine structure constant. For a typical $\mu_{e}(0)=$ $(10-20) \mathrm{MeV}$, the electrosphere is about $10^{3} \mathrm{fm}$ thick or more. The variation of $\mu_{e}$ across the electrosphere is shown in Fig. 1. The scattering of electrons in the electrosphere gives rise to bremsstrahlung photons. The

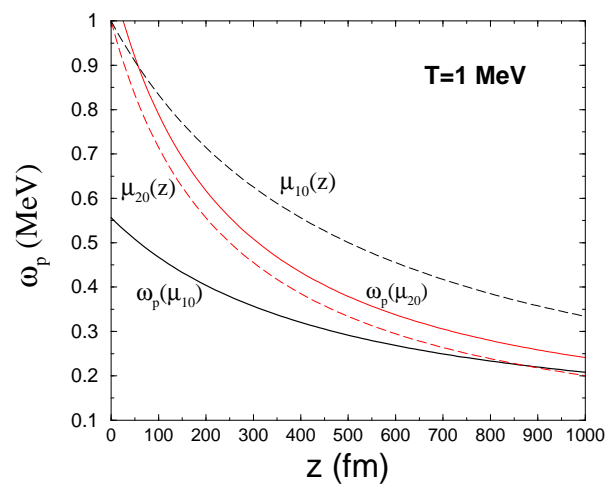

Figure 1. Profiles of the electron chemical potential $\mu_{e}$ (Eq. (2)) and the plasma frequency $\omega_{p}$ in the degenerate limit (Eq. (3)) versus distance $z$ from the surface of the strange quark matter star. $\mu_{10}\left(\mu_{20}\right)$ is the electron chemical potential in units of 10 (20) $\mathrm{MeV}$.

emitted photons have to travel through the electron plasma, therefore, their in-medium dispersion relation can be taken as $\omega=\left(\omega_{p}^{2}+k^{2}\right)^{1 / 2}$ (in $c=$ 1 units), where the plasma frequency $\omega_{p}$ serves as a low-energy cut-off. For degenerate electrons $\left(T / \mu_{e} \ll 1\right)$, the plasma frequency is determined from ${ }^{8}$

$$
\omega_{p}^{2} \cong \frac{4 \alpha}{3 \pi} \mu_{e}^{2}\left(1+\frac{\pi^{2} T^{2}}{3 \mu_{e}^{2}}\right) .
$$

The optical depth of the electrosphere at any distance is determined by $\omega_{p}$, which decreases with increasing $z$ (see Fig. 1). The emissivity $Q$ is thus a 
function of $\omega_{p}(z)$. The total luminosity can be expressed as

$$
L=4 \pi R^{2} \int_{z=0}^{z=z_{0}} Q\left(\omega_{p}(z)\right) d z,
$$

where $R$ is the star's radius and $z_{0}$ is the thickness of the electrosphere. As quark matter is admixed with electrons in the innermost region of the electrosphere, the exchanged photon is screened. We take this into account by modifying the photon propagator appropriately. Our results demonstrate that electric screening and magnetic damping effects play only a small role for the emission of low energy photons.

\section{Calculation of Photon Emissivity}

The photon emissivity from the bremsstrahlung process is

$$
\begin{aligned}
Q=\frac{2 \pi}{s \hbar} & \quad\left[\prod_{i=1}^{4} \int \frac{d^{3} p_{i}}{(2 \pi)^{3} 2 \omega_{p_{i}}}\right] \int \frac{d^{3} k}{(2 \pi)^{3} 2 \omega_{k}} \omega_{k} \sum_{\text {spin }}|M|^{2} S_{L P M}(k) \\
& \times n_{F}\left(\omega_{p_{1}}\right) n_{F}\left(\omega_{p_{2}}\right) \tilde{n}_{F}\left(\omega_{p_{3}}\right) \tilde{n}_{F}\left(\omega_{p_{4}}\right)(2 \pi)^{3} \delta^{3}\left(\mathbf{P}_{\mathbf{f}}-\mathbf{P}_{\mathbf{i}}\right) \delta\left(E_{f}-E_{i}\right) .
\end{aligned}
$$

The subscripts $i=1$ to 4 refer to electrons, $\left(\omega_{k}, \mathbf{k}\right)$ is the 4 -momentum of the emitted photon. ${ }^{a}$. The phase space for electrons is convoluted with the appropriate Fermi distribution functions $n_{F}\left(\omega_{p_{i}}\right)=1 /\left(\mathrm{e}^{\omega_{p_{i}} / T}+1\right)$ (in $k_{B}=1$ units) and $\tilde{n}_{F}=1-n_{F}$, respectively. Pauli blocking for degenerate fermions restricts the intermediate state to be almost on shell, so that the contribution of low-energy photons dominates the emissivity. $S_{L P M}$ is a suppression factor due to the Landau-Pomeranchuk-Migdal (LPM) effect of multiple scattering during emission, which we estimate numerically. For this section, we set $S_{L P M}=1$ to obtain useful analytical expressions for the emissivity. We work in Low's approximation which is accurate for low-energy photons. In this limit, the full scattering amplitude $M$ for bremsstrahlung factorizes into an elastic part (Møller scattering) and a part which gives the classical intensity divided by the energy $\omega_{k}$.

In the soft photon limit, the analytic expression for the emissivity $Q$ in two

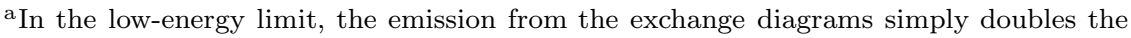
overall result for the emissivity from the direct diagrams (no interference). Since there is a symmetry factor of $s=2$ in the denominator of the emissivity expression Eq. (5), we may ignore the identity of the particles altogether and evaluate the emissivity from the direct diagrams alone.
} 
relevant regimes of temperature ${ }^{b}$ reads

$$
\begin{gathered}
Q=\frac{64 \alpha^{3} m_{e} \mu_{e}^{3} F\left(\omega_{p}\right)}{15 \hbar(2 \pi)^{6}} \ln \left(\frac{\mu_{e}}{m_{e}}\right) I\left(T, \mu_{e}\right) \\
F\left(\omega_{p}\right)=\left\{1+\frac{1}{2} \frac{\omega_{p}^{2}}{\omega_{p}^{2}+m_{e}^{2}}-\frac{3 \omega_{p}}{2 m_{e}} \tan ^{-1}\left(\frac{m_{e}}{\omega_{p}}\right)\right\} \\
I\left(T, \mu_{e}\right)=8 \pi^{2} \times\left\{\begin{array}{cc}
\frac{4 T}{\mu_{e}^{3}}\left(\ln 2-\frac{1}{2}\right) ; & \frac{m_{e}^{2}}{2 \mu_{e}} \leq T \ll \mu_{e} \\
\frac{2}{\mu_{e}^{2}} \mathrm{e}^{-m_{e}^{2} / 2 \mu_{e} T} ; \quad T \ll \frac{m_{e}^{2}}{2 \mu_{e}}
\end{array}\right\} .
\end{gathered}
$$

Above, terms of order $\left(m_{e}^{2} / m_{\text {Debye }}^{2}\right)$ arising from electric screening effects have been ignored because they are small. The factor $\ln \left(\frac{\mu_{e}}{m_{e}}\right)$ comes from forward scattering mediated by unscreened magnetic gluons. Thus, screening effects are sub-dominant in the low-energy limit. In order to test the accuracy of the analytic result, we performed a numerical integration of Eq. (5), and a comparison of analytical and numerical results, along with the pair-production rate ${ }^{10}$ is shown in Fig. 2.

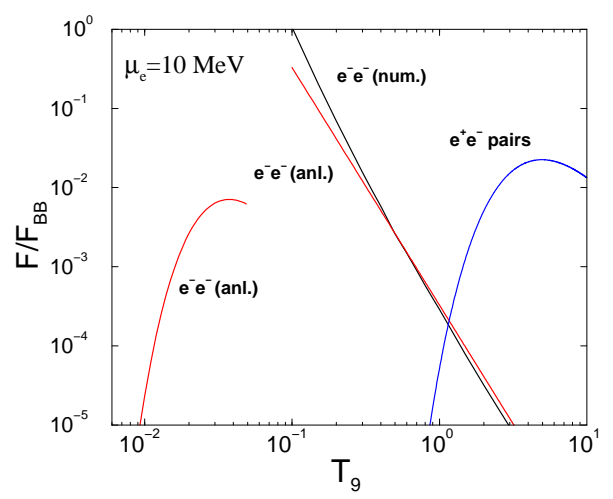

Figure 2. Photon flux from the bremsstrahlung and pair annihilation processes scaled to the blackbody limit as a function of temperature in units of $10^{9} \mathrm{~K}\left(T_{9}\right)$. The dashed curve (num.) shows results of numerical integrations of Eq. (5) which is to be compared with the results of the analytical expressions (anl.) from Eq. (6) for low and high temperatures. The (uniform) electron chemical potential is $\mu_{e}=10 \mathrm{MeV}$ with $z_{0}=100$ $\mathrm{fm}$.

${ }^{\mathrm{b}}$ When $T \sim m_{e}^{2} / \mu_{e}$, recoil effects become important and the matrix element is more complicated. 
The flux of bremsstrahlung radiation dominates over that from paircreation for electron plasmas below a temperature of $\sim 0.1 \mathrm{MeV}$. Its dependence on the third power of the electron chemical potential (or linear in electron density) distinguishes it from other processes.

The spectrum of low energy bremsstrahlung photons is given by ${ }^{9}$

$$
\omega_{k}\left(\frac{d \sigma}{d \omega_{k}}\right) \propto\left(1-\frac{\omega_{p}^{2}}{\omega_{k}^{2}}\right)^{3 / 2} \ln \left(\frac{\mu_{e}^{2}}{m_{e} \omega_{k}}\right) .
$$

In the quasiclassical approximation, support for this spectrum exists only between $\omega_{\min }=\omega_{p}$ and $\omega_{\max }=\left(\omega_{p}^{2}+m_{e}^{2}\right)^{1 / 2}$. One must go beyond this in order to obtain the spectrum for $\omega>\omega_{\max }$. Equation (7) shows that the presence of the electron plasma produces a harder spectrum than in vacuum, whereas the total photon flux is smaller. Although the contribution of high energy photons cannot be reliably estimated within our approximations, it is expected to fall off steeply due to the large electron degeneracy.
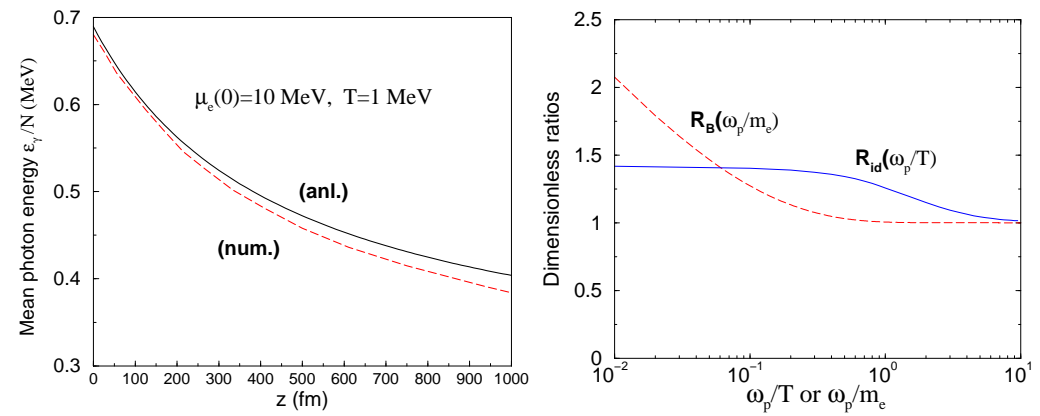

Figure 3. (left panel): Mean photon energy versus $z$, calculated analytically (anl) and numerically (num). (right panel): A comparison of the ratio $R$ in Eq. (9) for bremsstrahlung photons and $R_{i d}$ for thermal photons with in-medium photon mass $\omega_{p}$.

The mean photon energy from bremsstrahlung is given by (see Fig. 3)

$$
\langle\omega\rangle=Q / \Gamma \approx 0.5 \mathrm{MeV},
$$

where $\Gamma$ is the number of bremsstrahlung reactions per unit volume per unit time (the rate). Deviations from a blackbody spectrum are quantified by the dimensionless ratio

$$
R_{B}=\left\langle\omega^{2}\right\rangle /\langle\omega\rangle^{2},
$$


where $\left\langle\omega^{2}\right\rangle=Q^{2} / \Gamma$. Figure 3 contrasts this ratio for the bremsstrahlung spectrum against ideal gas behavior $R_{i d}$. The non-thermal feature of the bremsstrahlung photons is due to their large mean free paths in the electrosphere, so that they do not scatter often enough to thermalize. In light of the limited frequency support within our low-energy approximations, a more rigorous quantum calculation is called for to more accurately characterize the spectrum, which depends primarily on the ratio $\omega_{p} / m_{e}$.

Multiple scattering of electrons within the formation time of the radiated photon can lead to Landau-Pomeranchuk-Migdal (LPM) suppression of bremsstrahlung. A numerical integration of Eq. (5) including the suppression factor $S_{L P M}(k)$ reduces the simple analytical estimate of Eq. (6). Specifically, for $\mu_{10}=1$, the reduction factor (relative to the emissivity without the LPM effect) is $\sim 60$, while for $\mu_{10}=2$, the reduction factor is $\sim 350$. The mean photon energy and spectral shape do not change much upon the inclusion of the LPM suppression because both the rate and the energy loss are affected similarly, and nearly cancel in the ratio.

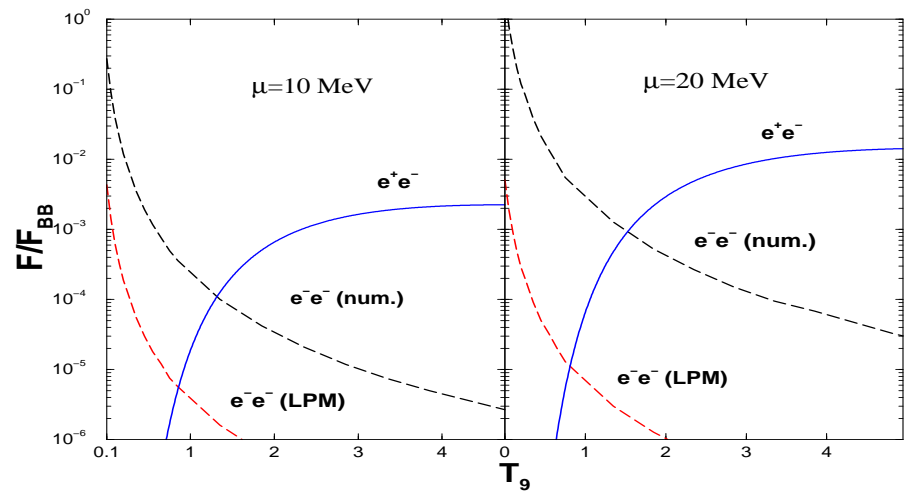

Figure 4. Photon flux from the bremsstrahlung and pair annihilation processes scaled to the blackbody value as a function of temperature in units of $10^{9} \mathrm{~K}\left(T_{9}\right)$. In the case of the bremsstrahlung process, the upper (lower) curve shows results without (with) the effects of the LPM effect.

\section{Importance for cooling of bare quark stars}

The emitted photons rob energy from the low temperature electron plasma and cause the surface of the quark star to cool. The bremsstrahlung rates calculated in this work, together with the rates of pair annihilation pro- 
cesses calculated earlier, can be used as input for calculations of the thermal evolution of strange quark matter stars. Improved estimates of the bremsstrahlung emissivity applicable in the temperature range excluded by Eq. (6) require a proper account of photon absorption processes, as bremsstrahlung emission becomes comparable to blackbody emission. However, for $T /{ }^{\circ} \mathrm{K} \leq 10^{9}$, bremsstrahlung is the dominant photon emission process, as the luminosity from pair annihilation is vanishingly small. The former also dominates equilibrium and non-thermal bremsstrahlung radiation from quark-quark collisions in the uppermost layer of quark matter ${ }^{11}$. As the temperature falls below $10^{7} \mathrm{~K}$, the rise in the bremsstrahlung rate is halted by the exponential factor in Eq. (6) which causes the emissivity to turn over and rapidly decrease. For $T<10^{9} \mathrm{~K}$, the bremsstrahlung process dominates photon emission even in the presence of LPM effects, suggesting that it will be important in baseline calculations of the surface luminosity (or temperature) versus age of bare quark stars that can help distinguish them from neutron stars.

\section{Acknowledgments}

P.J. and C.G. are supported in part by the Natural Sciences and Engineering Research Council of Canada and in part by the Fonds Nature et Technologies of Quebec. The research of M.P. was supported by the U.S. Department of Energy grant DOE/DE-FG02-87ER-40317 and the NSF

grant INT-9802680. The work of D.P. is partially supported by grants from UNAM-DGAPA (PAPIIT-IN112502) and Conacyt (36632-E).

\section{References}

1. Manju Prakash, E. Baron and M. Prakash, Phys. Lett. B243, 75 (1990).

2. E. Farhi and R. L. Jaffe, Phys. Rev. D30, 2379 (1984).

3. J. M. Lattimer and M. Prakash, Science 304, 536 (2004).

4. D. Page and V. V. Usov, Phys. Rev. Lett. 89, 131101 (2002).

5. See, e.g., J. Knodlseder, astro-ph/0207527.

6. M. Alford and K. Rajagopal, J. High Energy Physics, 0206031 (2002).

7. C. Kettner, F. Weber, M. K. Weigel and N. K. Glendenning, Phys. Rev. D51, 1440 (1995).

8. S. Ratković, S. I. Dutta and M. Prakash, Phys. Rev. D67, 123002 (2003).

9. P. Jaikumar, C. Gale, D. Page and M. Prakash, Phys. Rev. D, in press.

10. V.V. Usov, Astrophys.J. 550, L179 (2001).

11. K. S. Cheng and T. Harko, Astrophys.J. 596, 451 (2003). 